\title{
Monocilia in the embryonic mouse heart imply a direct role for cilia in cardiac morphogenesis
}

\author{
Jennifer Slough, Laura Cooney, and Martina Brueckner \\ Departments of Pediatrics and Genetics, Yale University School of Medicine, New Haven, CT \\ 06520 USA
}

\begin{abstract}
Primary cilia are required for signaling, chemosensing and mechanosensing in many fluid-filled organs, thus cilia could also have a direct role in heart development. They are essential to the development of cardiac LR asymmetry via their function at the embryonic organizer (node). We show that cilia are found in the mouse embryo heart at e9.5-e12.5. We demonstrate abnormal development of the endocardial cushions (ECCs) and compact myocardium (CM) in e9.5 mouse embryos with absent cilia. In contrast, hearts from embryos with abnormal LR development due to paralyzed, but structurally normal, node cilia show less severe ECC defects and normal CM. These observations suggest that a subset of cilia called cardiac cilia are required in cardiac development independently from their function in LR development. One possible function of cardiac cilia is as mechanosensors, integrating flow, cardiac function and morphogenesis.
\end{abstract}

\section{Keywords}

cilia; cardiac development

\section{Introduction}

Congenital heart disease is the most common serious birth defect, affecting $0.8 \%$ of liveborn infants. Normal cardiac development depends on complex interplay between genetic and epigenetic factors. Signaling pathways such as Wnt/ $\beta$-catenin and Hedgehog signaling, and cell behaviors such as epithelial-mesenchymal transformation (EMT) and neural crest cell migration are all essential in specifying heart morphogenesis (Dunwoodie, 2007). A unique feature of heart development is that the heart must function as a pump both to support the remainder of the developing organism, and to drive its own normal morphogenesis. Surgical and genetic manipulation in zebrafish demonstrated linkage between flow-mediated shear stress exerted on the endocardium and the formation of atrioventricular valves. Obstructing fluid flow early in cardiac development by obstructing the right ventricular outflow tract with a microbead results in failure to develop the ventricular chamber proximal to the obstruction, and failure to form valves (Hove et al., 2003). In zebrafish, the silent heart (sih, mutation in cardiac troponin $\mathrm{T}$ ) and cardiofunk (cfk, mutation in sarcomeric actin) mutations result in defects in myocardial function and impaired blood flow, and both fail to develop normal AV valves (Bartman et al., 2004). Also, valve development in zebrafish is disrupted by changes in ventricular geometry, which could secondarily affect blood flow (Scherz et al., 2008). Observations correlating cardiac function and morphogenesis have also been made in mammals. Mice with a mutation in the sodium-calcium exchanger have no

Corresponding Author: Martina Brueckner, Yale University School of Medicine, 333 Cedar Street, Fitkin 426, New Haven, CT 06520, 203-785-4765 (phone), 203-737-2286 (fax), martina.brueckner@yale.edu. 
heartbeat, although the hearts begin to loop, and looping is in the normal D-direction, no seeding of the ECCs occurs (Koushik et al., 2001). The commonality of these genetic and epigenetic manipulations is abnormal blood flow through the heart and vasculature at early developmental timepoints, and subsequent abnormal cardiac morphogenesis. If flow participates in driving the development of form in the developing heart (and vasculature) there must be a mechanism for the heart to sense and respond to fluid flow.

Since cilia are essential in a range of signaling pathways (Eggenschwiler and Anderson, 2007), and are the fluid-flow sensor in other organs consisting of fluid-filled lumens, such as the kidney, biliary tract and pancreas, they may also function in the developing heart. Cilia are found on human umbilical vein endothelial cells (HUVECs). The HUVEC cilia responded to increasing fluid flow by resorbing, thus providing the first evidence that cilia are found in vascular endothelium, and that they can respond to flow signals (Iomini et al., 2004). Little is known, however, about cilia in the developing heart. Monocilia have been identified in chicken embryonic hearts at HH stages 24-30 when atrial and ventricular septation and atrio-ventricular (AV) valve development is occurring. The monocilia were more prominent in areas of the heart corresponding to regions of low shear stress (Van der Heiden et al., 2006). Cilia can be classified as monocilia (also called primary cilia) and the classic cilia found on ciliated epithelia, such as the trachea. Monocilia are the single cilium found on almost every non-dividing cell, and are an important mechanism by which cells receive and transmit signals to the extracellular environment. Via the localization of a range of receptors on the ciliary membrane, and a variety of signaling molecules within the ciliary axoneme, they can function as chemosensors in the brain, light sensors in the retina, and mechanosensors in the kidney. While most monocilia are immotile, a specialized subset of monocilia contain axonemal dynein motors and are motile (Davis et al., 2006).

The LR asymmetry of the heart depends on global LR positional information generated by cilia during gastrulation. We have previously shown that the LR axis is initiated at the node of the mouse at e7.75. Two populations of node monocilia co-exist within the node (McGrath et al., 2003). The first are motile monocilia with a 9+2 microtubule structure (Caspary et al., 2007) containing the outer arm dyneins left-right dynein (lrd) (Supp et al., 1997) and DNAH5 (Olbrich et al., 2002). Coordinated beating of the collective motile node monocilia generates laminar leftward flow of the extraembryonic fluid surrounding the node, termed "nodal flow" (Nonaka et al., 1998). The second population of node monocilia has a 9+0 microtubule architecture, are immotile, and function as mechanosensors and/or chemosensors. They express the cation channel polycystin- 2 on the ciliary membrane, and respond to nodal flow by generating an increase in intracellular calcium in the cells at the left border of the node (McGrath et al., 2003). Thus, cilia serve multiple functions in the development of LR asymmetry. First, they are an inherently chiral structure, and thus function as the reference asymmetry in a bilaterally symmetric embryo. Second, they transmit this molecular asymmetry across the node region via leftward nodal flow. Finally, they sense nodal flow to convert the local nodal flow information to a broader domain of increased intracellular calcium.

Abnormal development of LR asymmetry results in failure of orientation of the heart loop along the LR axis. Therefore, some of the resulting complex cardiac defects associated are likely to be due to abnormal relative positioning of cardiac chambers and vessels in the setting of abnormal LR development. Mice and humans with immotile, but structurally normal node cilia have abnormal positioning of organs along the LR axis. This is associated with intracardiac defects in 40\% of affected mice (Icardo and Sanchez de Vega, 1991; Tan et al., 2007). Intracardiac defects are also seen in at least $6.5 \%$ of humans with immotile cilia (Kennedy et al., 2007). In contrast, mice with mutations resulting in either complete absence of cilia, or defective ciliary mechanosensation have $100 \%$ penetrance of severe 
intracardiac defects in addition to abnormal LR development. For example, mutation in the heterotrimeric kinesin components Kif3a or Kif3b results in a complete absence of cilia, and all affected embryos have intracardiac defects that lead to mid-gestational embryonic lethality independent of LR axis development. Mutation in Pkd2 results in defective ciliary mechanosensation and a $100 \%$ incidence of intracardiac defects such as atrio-ventricular canal defects (Nonaka et al., 1998; Marszalek et al., 1999; Wu et al., 2000; Boulter et al., 2001). This suggests that cilia have a much broader role in heart development via ciliary sensing and/or signaling than via their function in node ciliary motility alone.

In order to discern whether cilia have a role in cardiac morphogenesis beyond the establishment of LR asymmetry, we examined the hearts of the e9.5-e12.5 mouse embryo for the presence and distribution of cilia. We show that cilia are found on a wide range of surfaces, including those exposed to blood flow. Further, we show that the ECCs develop abnormally in hearts of mouse embryos mutant for heterotrimeric kinesin (Kif3a) and completely lacking cilia, and that the compact myocardium (CM) is thinned both in embryos mutant for Kif3a or polycystin2 (Pkd2), which functions as a mechanosensor in the kidney and node. This contrasts to the relatively normal hearts observed in embryos with immotile cilia due to mutation in left-right dynein (lrd) resulting in isolated LR defects. These data provide support for a direct role for cardiac cilia independent of their function in LR development, and suggests that they may be sensing blood flow and/or participating in other signaling pathways essential for cardiac morphogenesis.

\section{Results}

\section{Timing and distribution of cilia in embryonic mouse heart}

Any consideration of a direct function for cardiac cilia in heart development is based on finding cilia in the heart during embryogenesis, at time points critical for flow-directed cardiac morphogenesis. This corresponds to a time window extending from the onset of cardiac contraction at e 8.5 through the completion of valve formation and septation between e13.5 and e14.5. We evaluated the distribution of cilia in wild-type and Kif3a mutant embryo hearts by immunolabeling with antibodies against acetylated tubulin, which identifies cilia and other structures containing stable microtubules, such as the mitotic spindle and midbodies. As shown in Fig.1, cilia are distributed throughout the e9.5 embryonic mouse heart. At this stage, looping has occurred and the heart is contracting. Cilia line the endothelium of both the right and left atrial primordia (Fig.1C). Prominent cilia are also found on the endocardial layer surrounding the forming ventricular trabeculations (Fig.1D, Supplemental figure A). Fewer cilia are found in the early compact myocardial layer (data not shown). ECCs are beginning to form, and there are cilia on the endothelium at the atrial side of the ECCs (Supplemental figure B, red arrow), and on the mesenchymal cells within the ECCs (Supplemental figure B, yellow arrow). Cilia on the endocardial cells are predominantly located on the luminal surface, while cilia in the mesenchymal cells of the developing cushions are randomly oriented.

The e12.5 heart is shown in Fig.2. Atrial and ventricular septation is well underway. Prominent trabeculations have formed in both ventricles. ECCs have formed, and have clearly visible epithelial and mesenchymal components. Blood is visible in the heart (red cells autofluoresce, and are seen in panels B-E as combined red and green fluorescence.) There are a few cilia found on the atrial endocardial layer (yellow arrows in Fig.2C), however, they are not as prominent as at e9.5. Cilia persist on the ventricular surface of trabeculations in both ventricles (Fig.2D). In the ECCs, cilia are found on the endothelial surface (yellow arrows in Fig. 2E), but are now also visible in the mesenchymal cells (green arrows in Fig. 2E). A striking finding in the e 12.5 heart is that the epicardial layer, which 
has now ensheathed the heart, is prominently ciliated (white arrows in Fig.2C and Fig.2F). The epicardial cilia extend toward the pericardial space (Supplemental figure C).

\section{Mutation of Kif3a results in complete absence of cardiac cilia}

Kif3a is a component of the heterotrimeric kinesin complex, which comprises two motor subunits, Kif3a and Kif3b, and a non-motor KAP protein. Heterotrimeric kinesin has multiple functions, including organelle transport and anterograde neuronal transport (Yamazaki et al., 1995). In addition, heterotrimeric kinesin is essential for anterograde intraflagellar transport (IFT), and mice and cells lacking any component of heterotrimeric kinesin fail to assemble cilia, although they have normal centrioles and basal bodies (Nonaka et al., 1998; Marszalek et al., 1999; Takeda et al., 1999). Mutation of Kif3a or Kif3b results in multiple developmental defects including random development of LR asymmetry and embryonic lethality by e10.5. Analysis of hearts of e9.5 Kif $3 \mathrm{a}^{-/-} \mathrm{embryos}$ reveals a complete absence of cilia (Fig.3B, D) as compared to a wild-type litter mate (Fig. $3 \mathrm{~A}, \mathrm{C}$ yellow arrows). Other stable microtubule structures, such as midbodies and mitotic spindles are found in both wild-type and $\mathrm{Kif}^{-1-} \mathrm{a}^{--}$embryos, as indicated by the red arrows. When cardiac cilia are observed with higher magnification, Kif3a ${ }^{-/-}$embryos have normal centrioles, but no cilium extends from the centriole (Fig. 3E). This contrasts with the $2-5 \mu \mathrm{M}$ long cilia observed in the hearts of wild-type, $\mathrm{lrd}^{-/-}$and $\mathrm{Pkd}^{-/-}$embryos (Fig. 3E).

\section{Abnormal development of the endocardial cushions and compact myocardium is observed in embryos with mutations affecting ciliary biogenesis or mechanosensing}

The hearts of e9.5 wild-type, $\operatorname{lrd}^{\Delta \text { neo/ } / \Delta \text { neo }}\left(\operatorname{lrd}^{-/-}\right.$, absent node cilia motility), $\mathrm{Pkd}^{-{ }^{-/}}$ (defective ciliary mechanosensation), and $\mathrm{Kif3a}^{-/-}$(complete absence of cilia) embryos were examined by H\&E staining. Embryos were obtained prior to lethality for the Kif3a ${ }^{-/-}$and $\mathrm{Pkd}^{-/-}$genotypes, and the hearts were beating at the time of harvest. Transverse sections were obtained after fixation and paraffin embedding. Since Kif3a, Ird and polycystin-2 are all required for development of LR asymmetry, we needed to control for the effects of abnormal looping direction per-se. To this end, since these mutations all result in random direction of heart looping, only embryos with a D-Looped heart were analyzed. Mesenchymal cells were counted in three $15 \mu \mathrm{M}$ sections of each genotype: WT- 66cells/ section, $\operatorname{lrd}^{\Delta \text { neo/ }} \Delta$ neo 32 cells/section, $\mathrm{Pkd}^{-/-} 46$ cells/section. The compact myocardium was assessed at the ventricular apex in a section encompassing the ventricular inflow in 2 separate embryos for each genotype. The $\operatorname{lrd}^{\Delta \text { neo/ } / \text { neo }}$ heart looked grossly comparable to wild-type (Fig.4A). There is decreased cellularity of the ECC compared to wild-type (Fig. 4B), however, the trabecular and CM both appear normal, with the compact myocardium being 2-3 cell layers thick, similar to wild-type (Fig.4C). No pericardial effusion is observed in the $\operatorname{lrd}^{\Delta \text { neo/ } / \Delta \text { neo }}$ embryos. The heart of the Pkd2 $2^{--}$embryo shown also has a D-loop (Fig. 4A). The ECCs have decreased cellularity, observed over multiple sections (Fig.4B), and there is only a minimal pericardial effusion. Notably, however, the CM is markedly thinned compared to wild type (Fig.4C), varying from 1-2 cell layers. The most severe phenotype was observed in the Kif3 $\mathrm{a}^{-/}$embryos. Here, the heart is surrounded by a large pericardial effusion (Fig.4A). The ECCs are almost completely acellular (Fig.4B), and the CM is reduced to a single cell layer (Fig.4C). Higher-magnification examination of the compact and trabecular myocardium shows normal trabecular and compact myocardial layers (black arrows) in the wild-type and $\operatorname{lrd}^{\Delta \text { neo/ } / \Delta \text { neo }}$ embryos (Fig.5A, B), and there is no significant pericardial effusion (blue arrows in Fig. 5A, B). Although pericardium, epicardium, myocardium and endocardium are all found and appear grossly normal in the $\mathrm{Pkd}^{-/}{ }^{-}$embryos (Fig.5C), the compact myocardial layer is thinner, and in some regions is reduced to one-cell thickness. In the Kif3 $\mathrm{a}^{-/-}$embryos (Fig.5D), the distinction of flattened epithelial cells comprising the epicardial and endocardial layers from the myocardial cells is much less distinct. The compact myocardial layer is rarely more than one cell in thickness, 
and there is a massive pericardial effusion (blue arrows). Together, these data indicate that absent polycystin2-mediated ciliary mechanosensation affects both the formation of ECCs and the development of normal CM. The most severe cilia defect, manifested by a complete absence of cilia, has a severe effect on all aspects of cardiac morphogenesis.

\section{Discussion}

Cardiac defects are a prominent feature of mouse embryos with mutations affecting cilia.

Although most ciliary mutations result in failure to develop normal LR asymmetry, we show here that the cardiac defects observed in embryos with ciliary abnormalities extend beyond those resulting from abnormal LR development. Further, we find cilia in the embryonic heart at stages corresponding to cardiac morphogenesis. These data point to a subset of primary cilia called cardiac cilia that have a direct role in cardiac morphogenesis independent of their function in LR axis formation.

Cardiac cilia are found in the mouse embryo heart at a time during development when they could function as mechanosensors, sensing blood flow and/or cardiac function, and/or they could function in a range of other cilia-dependent signaling pathways in cardiac morphogenesis. At e9.5, the heart is contracting and there is directional blood flow, and this stage coincides with the formation of ECCs and the development of compact and trabeculated myocardium. Similar to observations previously made on chick embryos, we identified cilia on the endocardium lining the atrium, on the endocardium of the forming ventricular trabeculations and on the endocardium lining the atrial and ventricular sides of the ECCs. These could represent regions of relatively low shear stress, and it is possible that cilia respond to shear stress in the heart by resorbing, similar to what has been observed in cultured HUVEC cells. The distribution of cilia in the heart is also dynamic with respect to time. At e12.5 we observed persistence of cilia in the atrium and the ventricular trabeculations. However, at this stage when ECCs are extensively cellular, few cilia are seen on the endocardial surface of the ECCs, however, prominent cilia are found in the mesenchymal ECC cells. Finally, the epicardium, which has now ensheathed the heart, has extensive monocilia. Since these cilia do not extend into the lumen of the heart, they cannot sense blood flow. However, it is possible that epicardial cilia are also mechanosensors, and sense myocardial function by detecting contact between the epicardium and the pericardium.

In order to evaluate the direct role of cilia in cardiac morphogenesis, we analyzed the early cardiac phenotype of mice with a range of mutations affecting ciliary biogenesis and function. It is essential to distinguish the cardiac defects resulting from disturbed development of LR asymmetry, from those due to a direct function for cardiac cilia. The difficulty here lies with the fact that cilia are essential to the development of LR asymmetry, and almost all mouse mutations affecting ciliary function also disrupt LR development. It has long been assumed that the intracardiac defects observed in mice and humans with laterality disturbances result entirely from abnormal positioning of otherwise inherently normal cardiac primordia along the embryonic LR axis. Our observations however indicate that LR development per-se does not exclusively control cardiac morphogenesis: the early cardiac phenotypes observed in mice with a range of ciliary mutations correlated with the underlying ciliary defect, not with the direction of the heart loop.

The cardiac defects observed in the $\operatorname{lrd}^{\Delta \text { neo/ } \Delta \text { neo }}$ mouse embryos with structurally normal, but immotile node cilia are the least severe. Lrd is an axonemal dynein, and thus far the only identified function for axonemal dyneins is ATP-driven sliding of microtubules in cilia to power ciliary motility. Expression of lrd in the early embryo between e 7.5 and e 10.5 is at the node and in the neural tube, and lrd is never expressed in the heart (Supp et al., 1999). Dynein-driven ciliary motility at the node is essential for the generation of LR asymmetry, 
and therefore mice with mutation in axonemal dyneins lrd or Dnahc5 have random LR development. Although $40 \%$ of $1 \mathrm{ld}^{-/-}$or Dnahc $5^{-/-}$hearts have intracardiac defects such as DORV (double outlet right ventricle), TGA (Transposition of the great vessels) or ECC defects, the remaining $60 \%$ have structurally normal hearts. In addition, the intracardiac phenotype can be normal or abnormal in the setting of either situs solitus or situs inversus, again suggesting that the direction of heart looping alone does not determine the final cardiac phenotype. Taken together, these observations suggest that the requirement for dynein-generated ciliary motility in heart development is limited to the requirement for ciliary motility in LR development. It is notable that the $6.5 \%$ incidence of complex intracardiac defects observed in humans with immotile cilia (Primary Ciliary Dyskinesia, PCD) is even lower than that described in mice, although this may represent an underestimate due to intrauterine loss of severely affected fetuses, and limited clinical evaluation of ciliary function in patients with heterotaxy (Kennedy et al., 2007).

In contrast to absence of node ciliary motility, isolated absence of polycystin 2 mediated mechanosensation leads to a more severe cardiac phenotype, and complete absence of cilia as observed in the Kif3a ${ }^{-/-}$embryos generates the most severe cardiac phenotype. Unlike lrd, Kif3a is expressed ubiquitously, and Pkd2 is expressed in the heart at e8.5 through e15.5 (Guillaume and Trudel, 2000). This suggests that different ciliary functions are required at distinct stages of heart development, as outlined in Figure 6. First, at e8.0, a combination of ciliary motility and mechanosensation at the node establishes embryonic LR asymmetry. This asymmetry is signaled to the cardiac mesoderm via an as yet poorly understood mechanism. Later, between e 8.5 and e $12.5+$, cardiac cilia are required to directly influence cardiac morphogenesis. In this scenario, cardiac cilia would be expected only to participate in cilia-mediated signaling or to sense blood flow generated by cardiac contraction, and not generate flow through ciliary motility. Mutations affecting only ciliary motility exert their effect on heart development only indirectly via their effect on LR development, while mutations affecting ciliary structure and/or mechanosensation impact both LR development and later stages of cardiac morphogenesis.

The specific nature of the early cardiac structural defects found in the hearts of embryos with mutations affecting cilia may give some insight into the function of cardiac cilia. We observed a striking decrease in the cellularity of the ECCs in embryos with absent cilia. In particular, the Kif $3 \mathrm{a}^{-/-}$embryos had no mesenchymal cells in the ECCs, and the Pkd2 $2^{-/-}$ embryos also had a consistent decrease in the cellularity of their ECCs. It is interesting to note that ECC defects are the most common intracardiac defect in humans with heterotaxy. If regional flow differences are sensed by cilia to promote epithelial mesenchymal transformation (EMT) in specific regions of endocardium destined to become AV valves, then absence of flow should result in abnormal ECCs: indeed, mouse mutations that result in absent flow produce severe ECC abnormalities (Koushik et al., 2001). These data raise the possibility that cilia in the endocardium of the ECCs sense localized shear forces, and contribute to regional epithelial-mesenchymal transformation. Regions corresponding to relatively low shear stress, such as the atrial side of the ECCs retain cilia, which could signal to permit EMT. As cushion development proceeds, regions such as the central cushions experience higher shear stresses, leading to a loss of cilia and inhibition of EMT. In this model, mice with absent cilia would be expected to exhibit decreased EMT, which is supported by our observation that the Kif3 $\mathrm{a}^{-/-}$embryos have acellular cushions despite the presence of blood flow. The mild decrease in ECC cellularity observed in $\operatorname{lrd}^{-/-}$embryos may be a manifestation of abnormal flow resulting from subtle looping abnormalities (as lrd is not expressed in the heart at the time of cushion formation), and the final phenotype observed in embryos with ciliary abnormalities could represent a product of looping abnormalities, defective intracardiac cilia-mediated sensing and possibly secondary effects resulting from potential roles of cilia in vascular development. 
The defects observed in the embryos with Pkd2 mutation are less severe than those observed in the embryos with absent cilia, especially with respect to AV valve development. Since polycystins are primarily mechano/flow sensors in the kidney, and in other organisms such as C.elegans (Barr et al., 2001), the relatively mild early cardiac defects in the $\mathrm{Pkd}^{-{ }^{-}-}$ embryos suggest that cardiac cilia have functions in heart development beyond mechanosensation. Alternatively, it is possible that there is polycystin2-independent ciliary flow sensing in the heart.

In addition to ECC defects, the CM in embryos with absent ciliary biogenesis (Kif3a ${ }^{-/-}$) or ciliary mechanosensation $\left(\mathrm{Pkd}^{-/-}\right)$is thinner than that observed in either control or $\operatorname{lrd}^{\Delta \text { neo/ } \Delta \text { neo }}$ embryos. As cilia are observed in both the ventricular endocardium and the epicardium, several factors may contribute to the CM defects observed in the ciliary mutant hearts. Cilia are intimately connected with the cell cycle and regulation of proliferative responses (Pugacheva et al., 2007; Qin et al., 2007). In the ventricular chamber they may function as flow sensors, and potentially regulate proliferation of the myocardium in response to flow signals. Alternatively (or in addition), cilia located on the epicardium could act as mechanosensors by sensing contraction of the heart in the pericardial sac. Epicardium is involved in regulation of myocardial development, as evidenced by severe thinning of the myocardium observed in mice with targeted deletion of WT1 (Kreidberg et al., 1993), which is expressed abundantly in the epicardium, but not in the myocardium.

The role of cilia in cardiac morphogenesis is complex. First, there is the known indirect effect resulting from the role of cilia in LR development. In addition, however, we show here that cilia have other direct roles in cardiac morphogenesis: cilia may function as flow sensors in the heart and/or vasculature, they may be integral to the regional control of EMT in the ECCs, and they may also sense myocardial function in the ventricle and/or epicardium to regulate the proliferative response of the developing myocardium. In addition, it is notable that there is substantial overlap between the cardiac phenotype observed in the Kif3a mutants and in mice with mutation in Smoothened, an essential component of the hedgehog signaling pathway (Zhang et al., 2001). As cilia are essential in hedgehog signaling, cardiac cilia are potential mediators of hedgehog signals in cardiac development (Huangfu et al., 2003; Corbit et al., 2005). Finally, because structurally abnormal hearts are likely to have abnormal flow patterns, and the thinned myocardium observed in many of the ciliary mutations is likely to have altered contractile function, defective cilia may both initiate and propagate abnormal cardiac development. Further effort aimed at deletion of cilia from specific regions in the developing heart will help understand the mechanism by which cardiac cilia function in heart development.

\section{Experimental Procedures}

\section{Mice}

$\operatorname{lrd}^{\Delta \text { neo/ } \Delta \text { neo }}$ were generated by placing an in-frame fusion of GFP into the N-terminal of lrd, and placing the neomycin resistance gene flanked by loxP sites into the first intron of lrd. The resulting $\operatorname{lrd}^{\Delta \text { neo/ } / \Delta \text { neo }}$ mice have a high incidence of situs inversus and perinatal lethality, and due to a splice abnormality fail to make any full-length lrd message (McGrath et al., 2003). Kif3 $\mathrm{a}^{\mathrm{fl} / \mathrm{fl}}$ mice were obtained from Larry Goldstein (University of California, San Diego) (Marszalek et al., 2000), and Kif3a ${ }^{-/-}$mice were obtained by crossing Kif3 $\mathrm{a}^{\mathrm{fl} / \mathrm{fl}}$ mice with mice expressing Cre recombinase under the control of the actin promoter. $\mathrm{Pkd}^{-/}$ mice were obtained from Stefan Somlo (Yale University School of Medicine) (Wu et al., 2000). Embryos were obtained from timed intercrossing of heterozygous mice for Kif3a ${ }^{-/-}$ and $\mathrm{Pkd}^{-/-}$mice, and from homozygous crosses for $\operatorname{lrd}{ }^{\Delta \text { neo/ } / \Delta \text { neo }}$ mice. Noon of the day of detection of the vaginal plug was considered as $0.5 \mathrm{dpc}$. 
Histology

Wild-type and mutant embryos were fixed overnight in $4 \%$ buffered paraformaldehyde and then dehydrated, cleared, and paraffin wax embedded. Transverse sections $(15 \mu \mathrm{m})$ were stained with Hematoxylin and Eosin using standard histology techniques.

\section{Immunohistochemistry}

Wild-type and mutant embryos were fixed overnight in $4 \%$ buffered paraformaldehyde and then dehydrated, cleared, and paraffin wax embedded. Transverse sections $(15 \mu \mathrm{m})$ were obtained. The slides were cleared in xylene and rehydrated in a series of graded solutions of ethanol and water. They were washed in PBS before performing antigen retrieval (Signet Retrieve-All 2). The slides were blocked for 1 hour with 5\% normal goat serum in PBS. They were incubated for 2 hours at room temperature with a 1:200 dilution of mouse monoclonal anti-acetylated tubulin clone 6-IIB-1 (Sigma) in PBS-5\% goat serum, followed by four 20-minute PBS-0.1\% Tween 20 (American Bioanalytical) (PBT) at room temperature. They were then incubated for 45 minutes in a 1:200 dilution of Texas Redconjugated antimouse antibody in PBS-5\% goat serum, followed by four 20-minute washes in PBT at room temperature, adding Hoechst (Molecular Probes) at a 1:1000 dilution in wash three. The slides were rinsed in water and cover-slipped using ProLong Antifade medium (Molecular Probes). Sections were examined with a Zeiss Axiovert microscope equipped with Apotome imaging.

\section{Supplementary Material}

Refer to Web version on PubMed Central for supplementary material.

\section{Acknowledgments}

We would like to thank Svetlana Makova, Jeffrey Drozd, and James McGrath for their assistance with this project, Mustafa Khokha for critical reading of the manuscript as well as Larry Goldstein (UCSD) and Stefan Somlo (Yale) for their genetically modified mice.

Grant information: NIH RO1 HD045789 (M.Brueckner, PI).

\section{References}

Barr MM, DeModena J, Braun D, Nguyen CQ, Hall DH, Sternberg PW. The Caenorhabditis elegans autosomal dominant polycystic kidney disease gene homologs lov-1 and pkd-2 act in the same pathway. Curr Biol. 2001; 11:1341-1346. [PubMed: 11553327]

Bartman T, Walsh EC, Wen KK, McKane M, Ren J, Alexander J, Rubenstein PA, Stainier DY. Early myocardial function affects endocardial cushion development in zebrafish. PLoS Biol. 2004; 2:E129. [PubMed: 15138499]

Boulter C, Mulroy S, Webb S, Fleming S, Brindle K, Sandford R. Cardiovascular, skeletal, and renal defects in mice with a targeted disruption of the Pkd1 gene. Proc Natl Acad Sci U S A. 2001; 98:12174-12179. [PubMed: 11593033]

Caspary T, Larkins CE, Anderson KV. The graded response to Sonic Hedgehog depends on cilia architecture. Dev Cell. 2007; 12:767-778. [PubMed: 17488627]

Corbit KC, Aanstad P, Singla V, Norman AR, Stainier DY, Reiter JF. Vertebrate Smoothened functions at the primary cilium. Nature. 2005; 437:1018-1021. [PubMed: 16136078]

Davis EE, Brueckner M, Katsanis N. The emerging complexity of the vertebrate cilium: new functional roles for an ancient organelle. Dev Cell. 2006; 11:9-19. [PubMed: 16824949]

Dunwoodie SL. Combinatorial signaling in the heart orchestrates cardiac induction, lineage specification and chamber formation. Semin Cell Dev Biol. 2007; 18:54-66. [PubMed: 17236794]

Eggenschwiler JT, Anderson KV. Cilia and developmental signaling. Annu Rev Cell Dev Biol. 2007; 23:345-373. [PubMed: 17506691] 
Guillaume R, Trudel M. Distinct and common developmental expression patterns of the murine Pkd2 and Pkd1 genes. Mech Dev. 2000; 93:179-183. [PubMed: 10781953]

Hove JR, Koster RW, Forouhar AS, Acevedo-Bolton G, Fraser SE, Gharib M. Intracardiac fluid forces are an essential epigenetic factor for embryonic cardiogenesis. Nature. 2003; 421:172-177. [PubMed: 12520305]

Huangfu D, Liu A, Rakeman AS, Murcia NS, Niswander L, Anderson KV. Hedgehog signalling in the mouse requires intraflagellar transport proteins. Nature. 2003; 426:83-87. [PubMed: 14603322]

Icardo JM, Sanchez de Vega MJ. Spectrum of heart malformations in mice with situs solitus, situs inversus, and associated visceral heterotaxy. Circulation. 1991; 84:2547-2558. [PubMed: 1959204]

Iomini C, Tejada K, Mo W, Vaananen H, Piperno G. Primary cilia of human endothelial cells disassemble under laminar shear stress. J Cell Biol. 2004; 164:811-817. [PubMed: 15024030]

Kennedy MP, Omran H, Leigh MW, Dell S, Morgan L, Molina PL, Robinson BV, Minnix SL, Olbrich H, Severin T, Ahrens P, Lange L, Morillas HN, Noone PG, Zariwala MA, Knowles MR. Congenital heart disease and other heterotaxic defects in a large cohort of patients with primary ciliary dyskinesia. Circulation. 2007; 115:2814-2821. [PubMed: 17515466]

Koushik SV, Wang J, Rogers R, Moskophidis D, Lambert NA, Creazzo TL, Conway SJ. Targeted inactivation of the sodium-calcium exchanger (Ncx1) results in the lack of a heartbeat and abnormal myofibrillar organization. Faseb J. 2001; 15:1209-1211. [PubMed: 11344090]

Kreidberg JA, Sariola H, Loring JM, Maeda M, Pelletier J, Housman D, Jaenisch R. WT-1 is required for early kidney development. Cell. 1993; 74:679-691. [PubMed: 8395349]

Marszalek JR, Liu X, Roberts EA, Chui D, Marth JD, Williams DS, Goldstein LS. Genetic evidence for selective transport of opsin and arrestin by kinesin-II in mammalian photoreceptors. Cell. 2000; 102:175-187. [PubMed: 10943838]

Marszalek JR, Ruiz-Lozano P, Roberts E, Chien KR, Goldstein LS. Situs inversus and embryonic ciliary morphogenesis defects in mouse mutants lacking the KIF3A subunit of kinesin-II. Proc Natl Acad Sci U S A. 1999; 96:5043-5048. [PubMed: 10220415]

McGrath J, Somlo S, Makova S, Tian X, Brueckner M. Two populations of node monocilia initiate left-right asymmetry in the mouse. Cell. 2003; 114:61-73. [PubMed: 12859898]

Nonaka S, Tanaka Y, Okada Y, Takeda S, Harada A, Kanai Y, Kido M, Hirokawa N. Randomization of left-right asymmetry due to loss of nodal cilia generating leftward flow of extraembryonic fluid in mice lacking KIF3B motor protein. Cell. 1998; 95:829-837. published erratum appears in Cell 1999 Oct 1;99(1):117. [PubMed: 9865700]

Olbrich H, Haffner K, Kispert A, Volkel A, Volz A, Sasmaz G, Reinhardt R, Hennig S, Lehrach H, Konietzko N, Zariwala M, Noone PG, Knowles M, Mitchison HM, Meeks M, Chung EM, Hildebrandt F, Sudbrak R, Omran H. Mutations in DNAH5 cause primary ciliary dyskinesia and randomization of left-right asymmetry. Nat Genet. 2002; 30:143-144. [PubMed: 11788826]

Pugacheva EN, Jablonski SA, Hartman TR, Henske EP, Golemis EA. HEF1-dependent Aurora A activation induces disassembly of the primary cilium. Cell. 2007; 129:1351-1363. [PubMed: 17604723]

Qin H, Wang Z, Diener D, Rosenbaum J. Intraflagellar transport protein 27 is a small G protein involved in cell-cycle control. Curr Biol. 2007; 17:193-202. [PubMed: 17276912]

Scherz PJ, Huisken J, Sahai-Hernandez P, Stainier DY. High-speed imaging of developing heart valves reveals interplay of morphogenesis and function. Development. 2008; 135:1179-1187. [PubMed: 18272595]

Supp DM, Brueckner M, Kuehn MR, Witte DP, Lowe LA, McGrath J, Corrales J, Potter SS. Targeted deletion of the ATP binding domain of left-right dynein confirms its role in specifying development of left-right asymmetries. Development. 1999; 126:5495-5504. [PubMed: 10556073]

Supp DM, Witte DP, Potter SS, Brueckner M. Mutation of an axonemal dynein affects left-right asymmetry in inversus viscerum mice. Nature. 1997; 389:963-966. [PubMed: 9353118]

Takeda S, Yonekawa Y, Tanaka Y, Okada Y, Nonaka S, Hirokawa N. Left-right asymmetry and kinesin superfamily protein KIF3A: new insights in determination of laterality and mesoderm induction by kif3A- /- mice analysis. J Cell Biol. 1999; 145:825-836. [PubMed: 10330409] 
Tan SY, Rosenthal J, Zhao XQ, Francis RJ, Chatterjee B, Sabol SL, Linask KL, Bracero L, Connelly PS, Daniels MP, Yu Q, Omran H, Leatherbury L, Lo CW. Heterotaxy and complex structural heart defects in a mutant mouse model of primary ciliary dyskinesia. J Clin Invest. 2007; 117:37423752. [PubMed: 18037990]

Van der Heiden K, Groenendijk BC, Hierck BP, Hogers B, Koerten HK, Mommaas AM, Gittenbergerde Groot AC, Poelmann RE. Monocilia on chicken embryonic endocardium in low shear stress areas. Dev Dyn. 2006; 235:19-28. [PubMed: 16145662]

Wu G, Markowitz GS, Li L, D’Agati VD, Factor SM, Geng L, Tibara S, Tuchman J, Cai Y, Park JH, van Adelsberg J, Hou H Jr, Kucherlapati R, Edelmann W, Somlo S. Cardiac defects and renal failure in mice with targeted mutations in Pkd2. Nat Genet. 2000; 24:75-78. [PubMed: 10615132]

Yamazaki H, Nakata T, Okada Y, Hirokawa N. KIF3A/B: a heterodimeric kinesin superfamily protein that works as a microtubule plus end-directed motor for membrane organelle transport. J Cell Biol. 1995; 130:1387-1399. [PubMed: 7559760]

Zhang XM, Ramalho-Santos M, McMahon AP. Smoothened mutants reveal redundant roles for Shh and Ihh signaling including regulation of L/R symmetry by the mouse node. Cell. 2001; 106:781792. [PubMed: 11517919] 

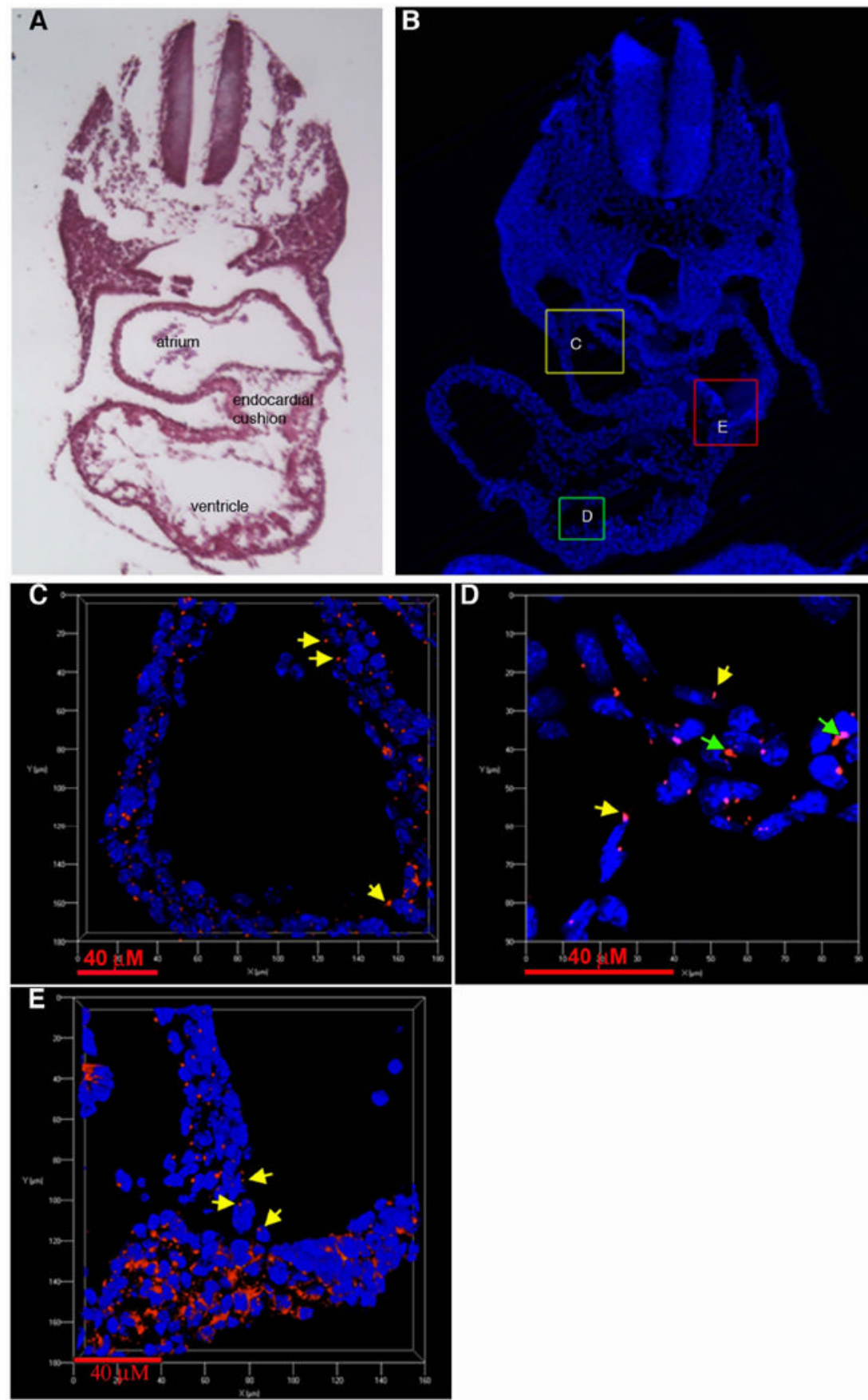

Figure 1.

Distribution of cilia in the e9.5 mouse embryo heart. A. e9.5 embryo cross section, H\&E stained. B. Hoechst (nuclear) fluorescence (blue), boxed outline panels enlarged in C-E. CE. Anti-acetylated tubulin labels cilia in red (yellow arrows), in addition to midbodies and mitotic spindles (green arrows). All images are a projection of Z-stacks taken through the $15 \mu \mathrm{M}$ section at a thickness of $.35 \mu \mathrm{M}$ C. Atrium. D. Trabeculations in left ventricle. E. Early ECCs, showing cilia on the atrial endothelial surface. 

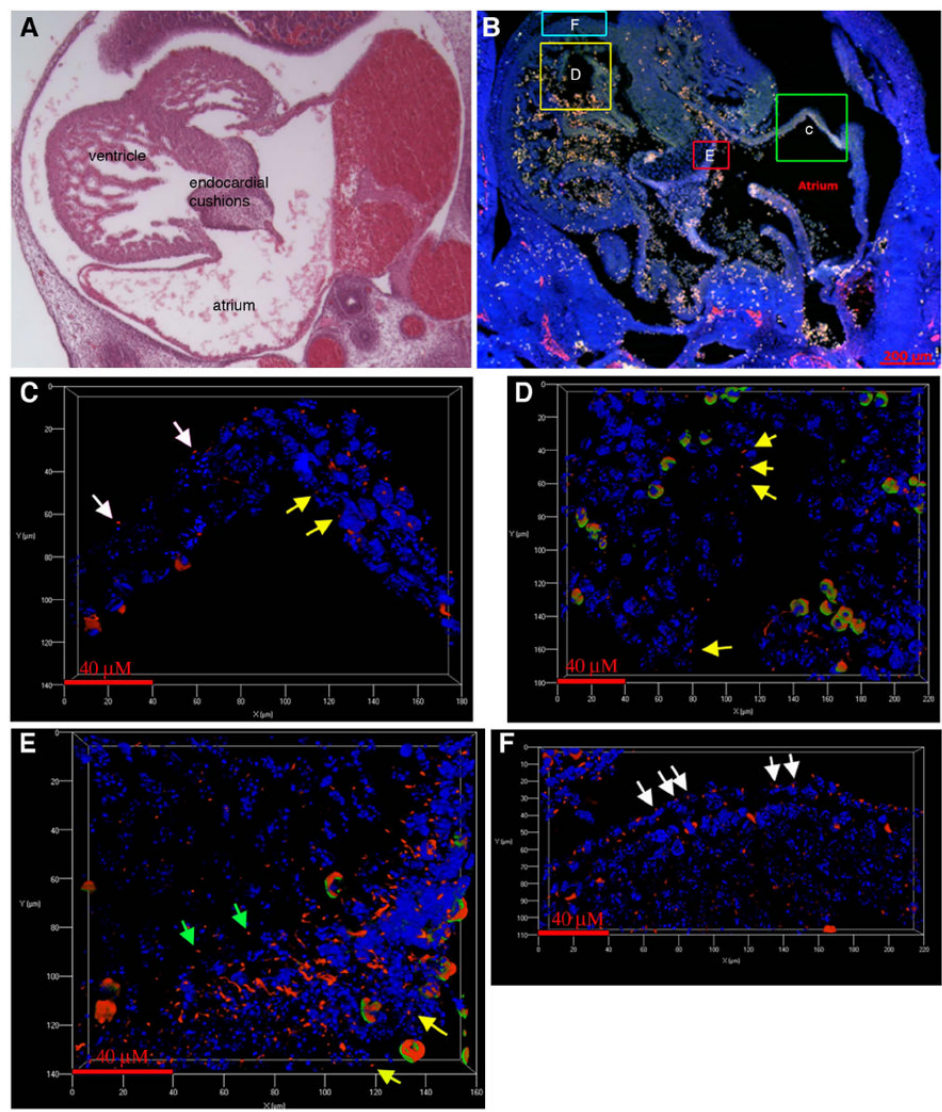

Figure 2.

Distribution of cilia in the e12.5 mouse embryo heart. A. e12.5 embryo cross section, H\&E stained. B. Hoechst (nuclear) fluorescence (blue), boxed outline panels enlarged in C-F. CF. Anti-acetylated tubulin labels cilia in red, red blood cells autofluoresce in green and red channels. All images are a projection of Z-stacks taken through the $15 \mu \mathrm{M}$ section at a thickness of $.35 \mu \mathrm{M}$ C. Atrium; cilia are on endocardial (yellow arrows) and epicardial (white arrows) surfaces. D. Ventricular trabeculations with cilia (yellow arrows). E. ECC; cilia are on endothelial surface (yellow arrows) and in mesenchymal cells (green arrows). F. Cilia on epicardial covering of ventricles (white arrows). 

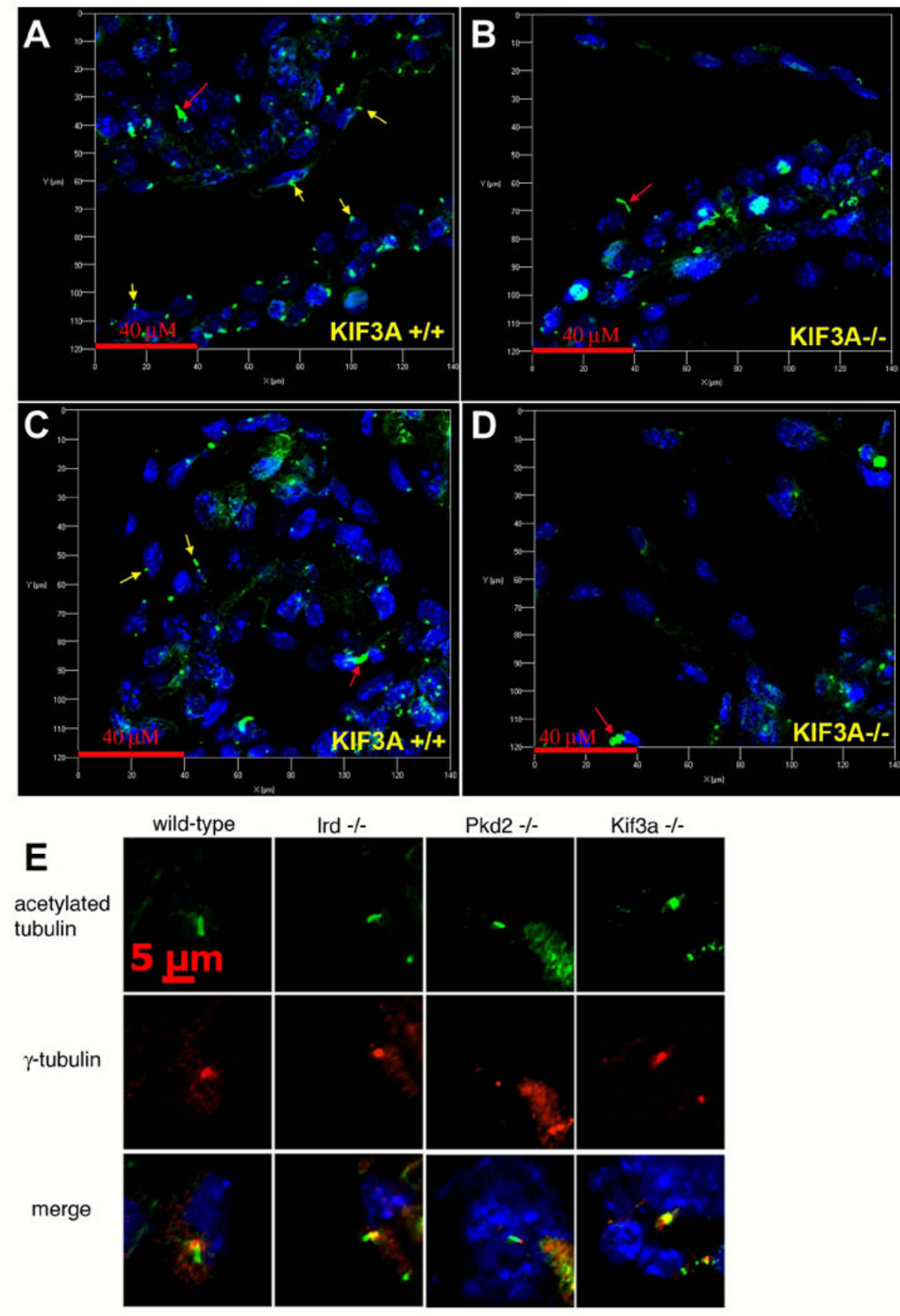

Figure 3.

Cilia are absent from the heart of e9.5 Kif3a ${ }^{-/-}$embryos. A-B. Myocardium, epicardium and pericardium of wildtype (A) and Kif3a $\mathrm{a}^{-/-}(\mathbf{B})$ hearts. Cilia (yellow arrows) are present in all 3 layers of the wild-type heart, and completely absent from the Kif3a ${ }^{-/-}$heart. Acetylated tubulin also identifies other stable microtubule structures, such as mitotic spindles and midbodies (red arrows); these are found in both wild-type and Kif $3 \mathrm{a}^{-/-}$hearts. C-D. Cilia are also found in the trabecular myocardium of wild-type hearts, but are absent in Kif $3 \mathrm{a}^{-/-}$ trabeculations. E. Cilia are normal in $\mathrm{Pkd}^{-/-}$and $\mathrm{lrd}^{-/-}$hearts, and absent in $\mathrm{Kif}^{-\mathrm{a}^{-/}}{ }^{-}$hearts. Centrioles are labeled with anti $\gamma$-tubulin in red, cilia with anti acetylated-tubulin in green. 
Wild-type, $\mathrm{Pkd} 2{ }^{-/}$and $1 \mathrm{rd}{ }^{-/-}$cilia extend $2-5 \mu \mathrm{M}$ from the centriole. No cilium is found extending from the centriole in Kif3 $\mathrm{a}^{-/-}$hearts. 


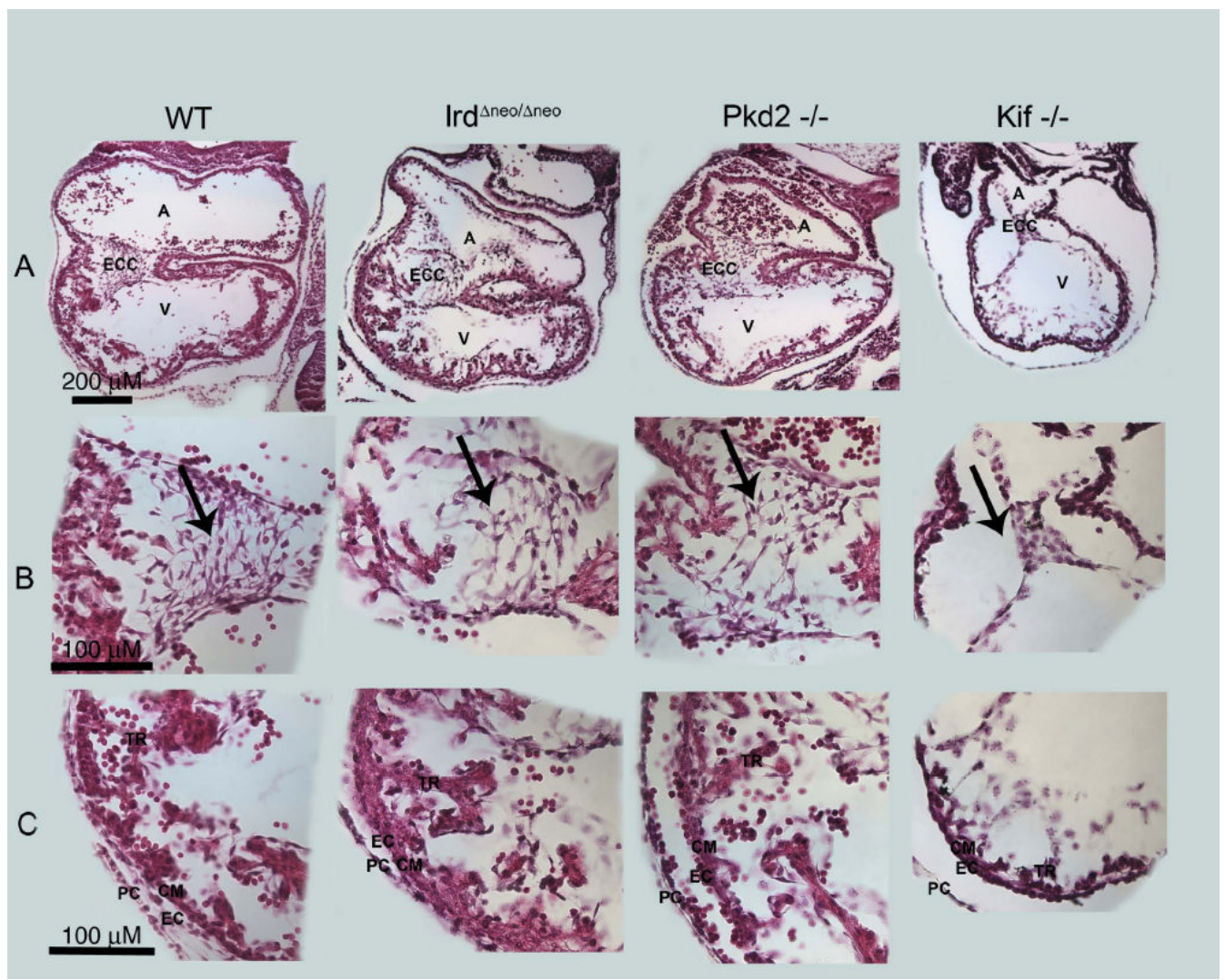

Figure 4.

Cross sections, H\&E stained, of the inflow (posterior region of the heart) of e9.5 wild-type, $\operatorname{lrd}^{\Delta \text { neo/ } \Delta \text { neo }}, \mathrm{Pkd}^{-/-}$and Kif3a $\mathrm{a}^{-/-}$embryos embryos. A. Overview images of wild type, $\operatorname{lrd}^{\Delta \text { neo } / \Delta \text { neo }}, \mathrm{Pkd}^{-/-}$, and $\mathrm{Kif} \mathrm{a}^{-/-}$hearts including the atrium (A), endocardial cushions $(\mathrm{ECC})$, and ventricle $(\mathrm{V})$. B. Endocardial cushions, arrow indicates cushion mesenchyme. $\mathbf{C}$. Higher-magnification view of the trabecular and compact myocardium (indicated by arrow), and the surrounding pericardium showing trabeculations in the WT and $\operatorname{lrd}^{\Delta \text { neo/ }} \Delta$ neo sections, decreased trabeculations in the $\mathrm{Pkd}^{-{ }^{-/}}$section, and none in the $\mathrm{Kif}_{3} \mathrm{a}^{-/-}$section. 


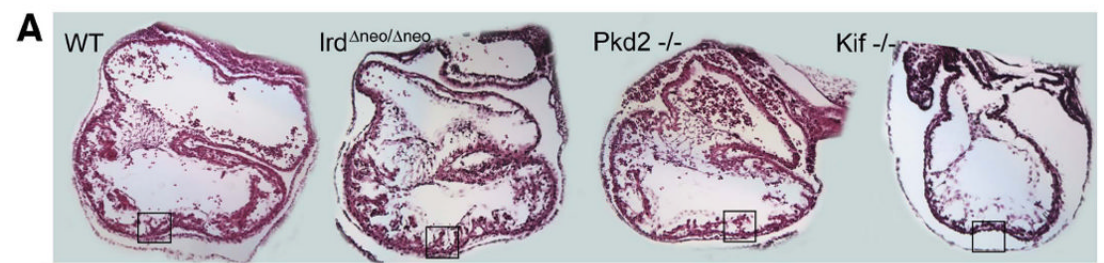

\section{B WT}
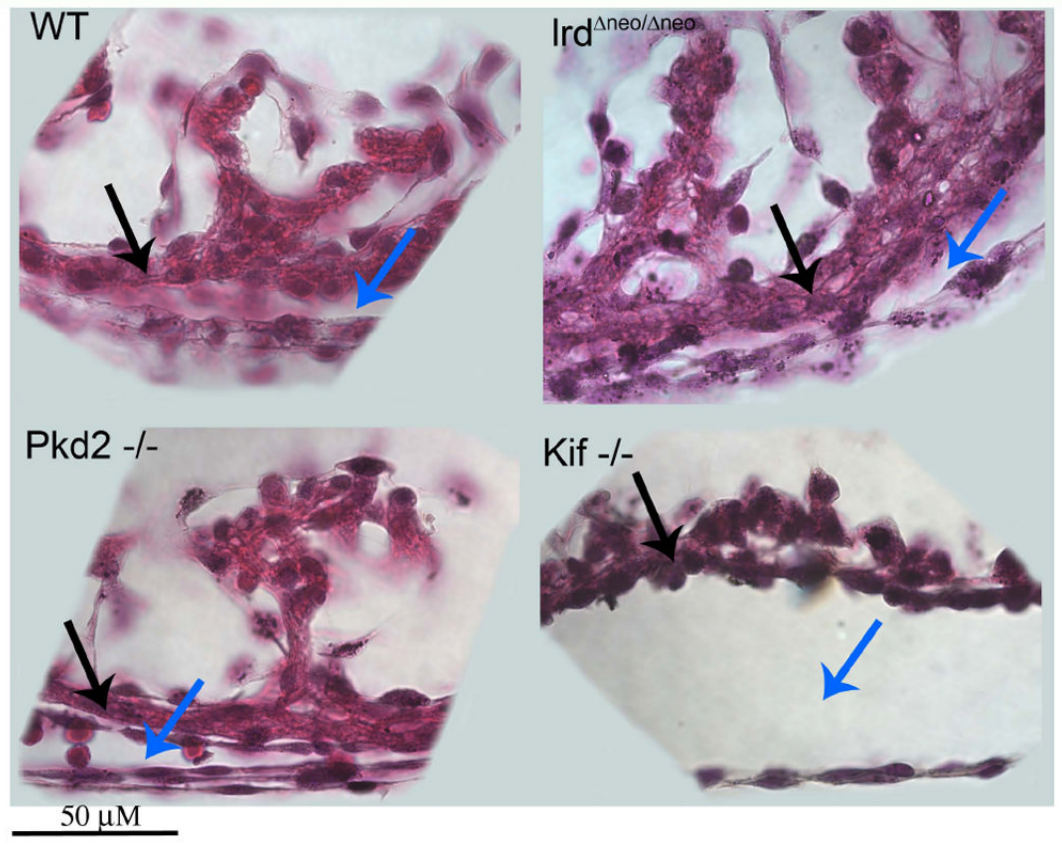

Figure 5.

High-magnification images of myocardium in e9.5 wild type, $\operatorname{lrd}{ }^{\Delta \text { neo/ } / \Delta \text { neo }}, \mathrm{Pkd}^{2 /-}$, and Kif $3 \mathrm{a}^{-/}$hearts. A. Overview, magnified areas are indicated by the black boxes. B. Highmagnification images, the compact myocardium is indicated by the black arrow and the pericardial space is indicated by the blue arrow. 


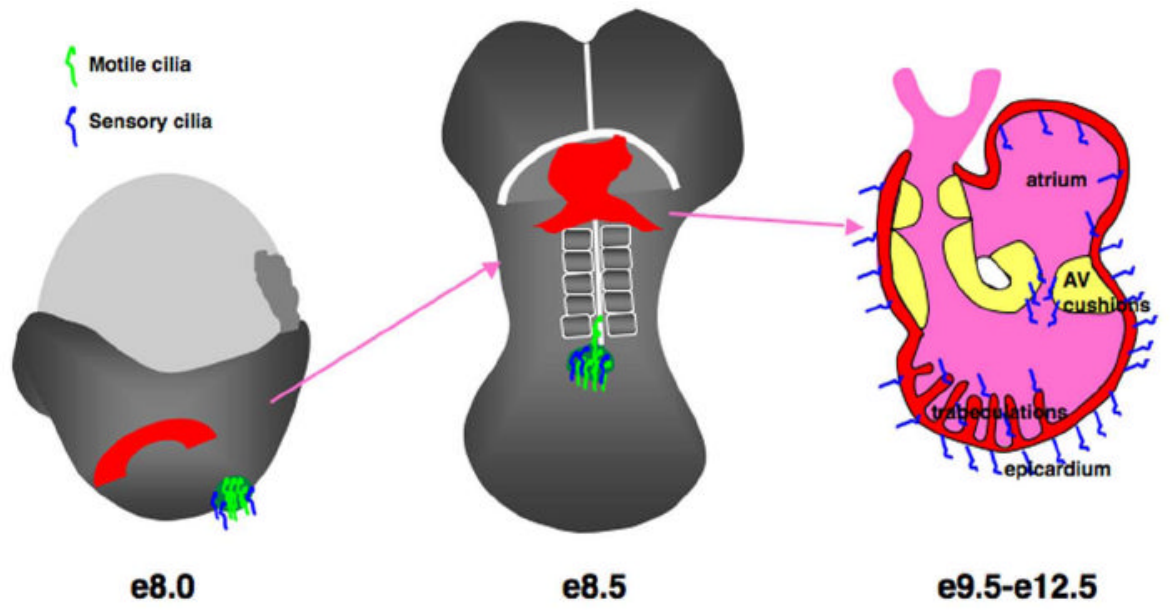

Figure 6.

Schematic for the function of cilia in heart development. At e 8.0, motile (green) and mechanosensory, immotile (blue) cilia establish embryonic LR asymmetry. At e9-e12+ mechanosensory cilia in the heart have a direct function in cardiac morphogenesis. 